\title{
Limits of drag augmentation at spacecraft end-of-mission and a mitigation strategy
}

\author{
Emma Kerr, Malcolm Macdonald $(\bowtie)$
}

University of Strathclyde, Glasgow G1 1 XJ, UK

\begin{abstract}
An increasing number of objects are being launched into low-Earth orbit. Consequently, to avoid the possibility of future in-orbit collisions space object removal techniques are receiving attention. As one of the most developed techniques, drag augmentation is increasingly being considered as an option for end-of-mission removal of objects from lowEarth orbit. This paper highlights a common misconception around drag augmentation: although it can be used to reduce de-orbit time, when used inappropriately it can increase the volume swept by an object and, thus, increase the occurrence risk of collision with another space object. Knowingly ignoring this increased risk of collisions could leave spacecraft operators, and consequently their responsible state party, open to liability risk. By investigating the volume swept and de-orbit lifetime, a strategy of delayed deployment is proposed as a compromise between reducing volume swept and time to de-orbit. However, this increases system complexity and, likely, cost.
\end{abstract}

\section{KEYWORDS}

drag augmentation

volume

area-time-product

space debris

liability

Learned Hand formula

calculus of negligence

\section{Research Article}

Received: 6 April 2020

Accepted: 11 August 2020

(C) The Author(s) 2020

\section{Introduction}

In January 2007, China conducted a direct-ascent antisatellite test, destroying the $750 \mathrm{~kg}$ Chinese weather satellite FY-1C (COSPAR identification 1999-025A) at an altitude of $865 \mathrm{~km}$ using a kinetic kill vehicle traveling in the opposite direction [1]. Whilst not the first, nor most recent such test in space, the altitude was higher than prior Russian and US tests, and a more recent Indian test, creating a prolonged and dispersed debris cloud that has had a significant impact on the space debris environment in low-Earth orbit (LEO). In February 2009, the defunct Cosmos-2251 (COSPAR identification 1993-036A) and active Iridium-33 (COSPAR identification 1997-051C) satellites collided at an altitude of $789 \mathrm{~km}$ [2], the first observed hypervelocity collision between two artificial satellites, leading to further growth in the LEO debris population. These two events have significantly increased awareness of the challenge presented by space sustainability. Meanwhile, the number of spacecraft launched per year has recently and rapidly increased. This trend is a result of the increased use of standardized, small and micro-satellite, platforms such as the CubeSat [3,4]. As

凶 Malcolm.macdonald.102@strath.ac.uk such, the prudency of space debris mitigation standards and regulations means space actors are increasingly implementing end-of-mission disposal plans to be, and be seen as, responsible and sustainable actors.

No international treaty exists to specifically deal with the issue of space debris. However, both the Outer Space Treaty and the Liability Convention address liability issues by creating a fault-based liability for damage caused in space [5-7]. In addition, organizations, such as the Inter-Agency Space Debris Coordination Committee and the International Organization for Standardization (ISO), have developed best-practice guidelines $[8,9]$. These guidelines define two protected regions: LEO (defined as the region below $2000 \mathrm{~km}$ altitude, which is the area of interest herein) and geosynchronous Earth orbit (defined as the segment of the spherical shell from $200 \mathrm{~km}$ below to $200 \mathrm{~km}$ above the geostationary altitude, approximately $35,786 \mathrm{~km}$, and from $-15^{\circ}$ to $15^{\circ}$ of latitude). Both organizations focus on two major areas of space debris mitigation. They discuss avoiding both the intentional release of debris during nominal operations and unplanned spacecraft break-up. Secondly, they discuss 
the post-mission disposal of spacecraft, which is the area of interest herein. Best-practice guidelines currently recommend that post-mission disposal should result in permanent removal from the LEO-protected region within 25 years of decommissioning.

Although debris mitigation guidelines specify a time period within which spacecraft should be removed, the method of removing spacecraft is not specified. Many de-orbit concepts exist [10]. However, very few of these concepts are currently viable. One viable technique is drag augmentation, commonly referred as a drag sail; it requires the introduction of a large projected area perpendicular to the velocity vector to increase the effect of atmospheric friction (colloquially termed atmospheric drag), thus increasing the instantaneous area for possible collisions. Such concepts have received notable attention, with various funding bodies and licensing authorities (state parties) supporting technology and flight demonstrationse [11-18], as well as in the specialist and popular mediae [19-21].

Prior studies of the application of drag augmentation lack a full analysis of the implications of increasing projected area on collision risk, focusing principally on time to de-orbit and assuming a direct correlation with collision risk. For example, in Ref. [15], the effect of the solar cycle on de-orbit time is presented; however the effect on volume swept is not. Meanwhile, in Refs. [15, 22], the effect of increasing the projected area is briefly considered through what is termed the area-time-product (ATP), which is equivalent to volume swept, with both only considering results where deployment coincides with solar maximum; concluding that ATP and hence risk is reduced. This is shown in this paper to be correct, but to also be a best-case analysis when the effect of the solar cycle on volume swept is presented, closing a gap in the literature.

Although by no means the sole metrics, the simplest metrics available to quantify the collision risk are the amount of time an object spends on-orbit, and how large a volume it sweeps through in that time. Practically, both the time to deorbit and the volume swept are metrics for the probability of collision occurrence. A third metric for risk could also be considered: the composition of the object. Such a metric attempts to capture the probable result of a collision, thus focusing on the risk that the collision poses to the space environment. For example, if an object collides with the thin film of a drag sail it is less likely to be catastrophically damaged than if it collides with the main body of a spacecraft, and that film is unlikely to fragment into many parts. This logic is applied in Refs. [15,22] where the analysis seeks to minimize the further generation of debris rather than the total collision risk. This suggests that the drag sail surface area should be neglected from the ATP, that is, the volume swept analysis. However, fault liability results from any collision no matter the impact and as such the onus should be to minimize the collision risk and to do no harm, rather than trying to minimize further damage, which implies the concept of an allowable collision. Similar arguments are explicitly made elsewhere in the literature in favor of allowable collisions [23], rather than seeking to $d o$ no harm, and overlook the regulatory consequence of fault liability. However, this type of analysis can provide complementary insights to those presented herein. The level of liability resulting from a collision is a consequence of the level of damage. Therefore, this study focuses solely on the risk of any collision occurrence and, hence, the operators being liable for the consequences. In particular, it does not aim to address the consequences of a collision nor the level of liability incurred. The concept of a population weighted volume swept is introduced to better determine the collision risk, but the resulting liability from a collision is beyond the scope of this study.

The typical assumption is that the less time an object is on-orbit, the lower the collision risk, and as such increasing the area of a spacecraft will always be beneficial. This assumption will be shown to be misguided. Rather, by increasing the area of a spacecraft at the end of its mission an operator could on-occasion be argued at fault, and hence liable for any subsequent on-orbit collision.

\section{Method}

The volume swept and de-orbit lifetime (total time spent in orbit after spacecraft operations cease) are dependent on the mass and projected area of a spacecraft. Using a validated general perturbations method for orbit lifetime analysis, the relationship between mass, projected area, volume swept, and orbit lifetime can be demonstrated. In this study, the general perturbation method developed by Kerr and Macdonald is further developed [24]. This method was validated using historical two-line element data for twenty-one spacecraft with drag coefficients, surface areas, masses, and so forth taken from referenced 
Table 1 Algorithm used to calculate volume swept

\begin{tabular}{cl}
\hline Number & Step \\
\hline 1 & Calculate the orbital lifetime using Eq. (1) or (3), as appropriate. \\
2 & Calculate orbit period hence and semi-major axis after one revolution. \\
3 & Calculate the distance travelled along the orbital path in that single revolution by approximating it as a closed \\
& elliptical orbit, with an average semi-major axis, $a=\left(a_{0}-a_{1}\right) / 2$. \\
5 & Repeat steps $1-3$ until de-orbit is complete, defined herein as an altitude of $65 \mathrm{~km}$ as per Ref. [24]. \\
& Calculate the sum of the distances calculated in step 3 and multiply that sum by the projected area to attain \\
& the total volume swept.
\end{tabular}

sources. Where a specific value could not be sourced through reference, the recommended ISO value or method to estimate a value was applied [25]. This validation found the method gave an average orbit lifetime error of $3.5 \% \pm 3.25 \%$ [24]. It showed a better performance compared to third party tools: Systems Tool Kit from Analytical Graphics*, General Mission Analysis Tool from $\mathrm{NASA}^{\dagger}$, and Semi-Analytical Tool for End of Life

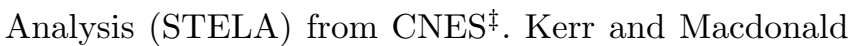
found STELA to be the most effective among the thirdparty tools, with an average orbit lifetime error of $6.63 \%$ $\pm 7.00 \%$ [24]. The Kerr-Macdonald method includes the effect of the solar activity cycle, thus capturing the time variance of the atmosphere required herein. As per Refs. [24], the orbit lifetime of a low eccentricity $(e<0.02)$ satellite is calculated as

$$
\tau_{\mathrm{L}}=\frac{e_{0}^{2}}{2 B^{\prime}}\left\{1-\frac{H}{a_{0}}\left[5+\frac{11}{20}\left(\frac{a_{0} e_{0}}{H}\right)^{2}\right]\right\}
$$

where $e$ is eccentricity, $a$ is semi-major axis, and $H$ is scale height. $B^{\prime}$ is calculated as

$$
B^{\prime}=\frac{2 \pi}{T_{0}} \frac{F S C_{\mathrm{D}}}{m} \rho_{0} a_{0} e_{0} \boldsymbol{I}_{1}\left[\frac{a_{0} e_{0}}{H}\right] \exp \left(-\frac{a_{0} e_{0}}{H}\right)
$$

where $T$ is the orbit period, $F$ is a factor considering the rotation of the atmosphere, $S$ is the projected area of the spacecraft in the instantaneous direction of travel, $C_{\mathrm{D}}$ is the drag coefficient of the spacecraft, $m$ is the mass of the spacecraft, $\rho$ is the atmospheric mass density, and $\boldsymbol{I}_{1}$ is the integrated form of the modified Bessel function [24]. Alternatively, if an orbit is approximately circular $(e<0.001$, as validated in Ref. [24]), the orbit lifetime can, with no loss in accuracy, be obtained as

\footnotetext{
* STK can be downloaded from http://www.agi.com/products/ stk/.

$\dagger$ GMAT can be downloaded from http://gmatcentral.org/.

$\ddagger$ STELA can be downloaded from https://logiciels.cnes.fr/ content/stela.
}

$$
\tau_{\mathrm{L}}=\frac{H T_{0}}{2 \pi \rho_{0} a_{0}^{2}} \frac{m}{F S C_{\mathrm{D}}}\left(1-\mathrm{e}^{\frac{a_{0}}{H}\left[\left(\frac{T_{\mathrm{f}}}{T_{0}}\right)^{\frac{2}{3}}-1\right]}\right)
$$

Here, subscript 0 denotes initial state, while subscript $\mathrm{f}$ denotes final state. To calculate volume swept, the full deorbit phase should be split into individual orbital revolutions, allowing the calculation of distance travelled. Using Eqs. (1)-(3), the volume swept can be calculated using the algorithm in Table 1.

Introducing a large surface area is frequently held to be beneficial as it lowers the orbit lifetime, and hence collision risk. However, using the volume swept as a metric for collision risk it can be shown that this presumed reduction in risk is not always the case. If the time-variant nature of the Earth's atmosphere is ignored, the volume swept is found to be directly proportional to mass and independent of the area projected to the atmosphere. Meanwhile, the orbit lifetime is not independent as it is inversely proportional to the projected area. Thus, the larger the spacecraft projected area, the shorter the lifetime, whilst volume swept remains constant. With the assumption of a time-invariant atmosphere, as has been widely applied in such prior studies, using volume swept and orbit lifetime as the measure of collision risk means drag augmentation is always beneficial in terms of debris mitigation measures. However, the Earth's atmosphere is highly dynamic and time-variant, and the effect of these variations must not be neglected [7]. To demonstrate the effect of the variation in mass and projected area over a de-orbit, these parameters are varied between 1 and $1000 \mathrm{~kg}$, and 0.01 and $50 \mathrm{~m}^{2}$, respectively. Two atmospheric models were also considered. The first uses a spherically-symmetrical, time-invariant atmosphere model with average solar activity developed in Ref. [24]. The second includes time-variance in that atmosphere model by incorporating the model of the solar activity cycle developed in Ref. [24]. The resultant volumes swept are shown in Fig. 1. The orbit used is cir- 

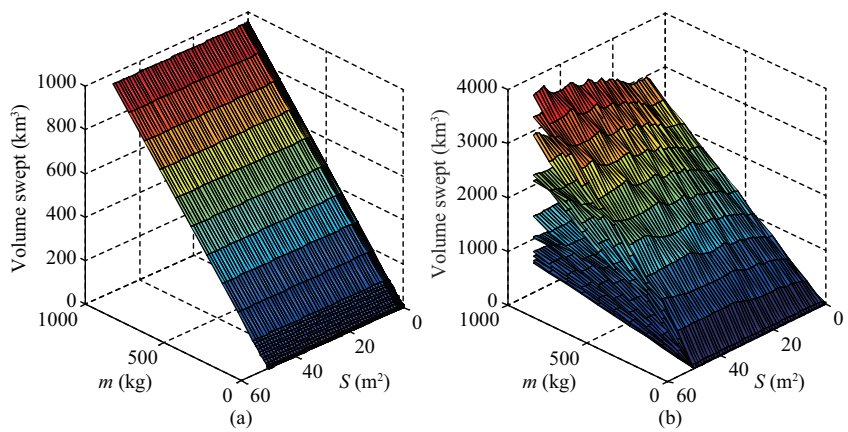

Fig. 1 Volume swept vs. mass $(m)$ and projected area $(S)$. Single surface in (a) shows the time-invariant atmosphere case. A total of 11 surfaces in (b) demonstrate a time-variant atmosphere case; each surface created using a different start date at 1-year intervals through the solar activity cycle.

cular with $65^{\circ}$ inclination and $400 \mathrm{~km}$ altitude, with orbit decay having occurred at $65 \mathrm{~km}$. Figure 1(a) shows the time-invariant atmosphere, and Fig. 1(b) shows a series of different start dates at 1-year intervals through the solar activity cycle, and thus 11 surfaces are presented. The solar activity cycle is the approximately 11-year variation in the amount of radiation that impacts Earth's atmosphere from the Sun. Increased radiation heats the atmosphere causing expansion, thus increasing atmospheric density at a given altitude [7]. Atmospheric density at altitudes of 100-1000 km during solar activity maximum can vary by up to two orders of magnitude from the minimum solar activity conditions [7]. Including this variation in atmospheric density means that spacecraft orbit lifetime, and hence volume swept, become dependent on the date at which de-orbit begins, as can be seen in Fig. 1.

As shown in Fig. 1(b), for the same mass and area, large differences can exist in the volume swept by a spacecraft depending on the start date through the solar activity cycle. This variation is not captured by considering orbit lifetime alone. It is of note that in this study it is assumed that the drag augmentation device is deployed to the required size, the nature and operations of this device are not considered and the device could be, for example, a drag sail, or balloon type device. Of note, Ref. [26] addresses the likelihood of achieving aerodynamic stability with a drag sail.

Given the large variation introduced by the start date during the solar cycle, a new de-orbit scheme is immediately apparent; taking advantage of the periods where a low volume swept occurs. By delaying deployment of the large surface area of a drag device to coincide with a period of high solar activity, and hence high drag, the volume swept by an object and its orbit lifetime can be optimized. However, the engineering implementation of this method, for example, maintaining compliance with spacecraft pacification guidelines, is beyond the scope of this study. The volume swept with the "delayed deployment" of a drag augmentation device can be calculated with a few minor additions to the algorithm presented in Table 1, as shown in Table 2. Further details of this scenario is discussed in the Results section.

End-of-mission is used in this paper to denote the point at which the spacecraft has completed its principal purpose, its mission; this can occur as a planned event or due to an anomaly on-board the spacecraft. At end-of-mission, the spacecraft is, wherever possible, decommissioned, within the context of this paper this will ideally include pacification of the spacecraft as per best-practice guidelines to avoid an unplanned spacecraft break-up [8,9], and triggering of the drag augmentation device, which may deploy immediately or at some planned time in the future. The de-orbit phase is defined

Table 2 Algorithm used to calculate volume swept with "delayed deployment" of a drag augmentation device

\begin{tabular}{cl}
\hline Number & Step \\
\hline 1 & Calculate the orbital lifetime using Eq. (1) or (3), as appropriate. \\
2 & Calculate orbital period and hence semi-major axis after one revolution. \\
3 & Calculate the distance travelled along the orbital path in that single revolution by approximating it as a closed \\
& elliptical orbit, with an average semi-major axis, $a=\left(a_{0}-a_{1}\right) / 2$. \\
4 & Repeat steps $1-3$ until de-orbit is complete, defined herein as an altitude of $65 \mathrm{~km}$ as per Ref. [24]. \\
5 & Calculate the sum of the distances calculated in step 3 prior to deployment of drag augmentation device and \\
& multiply that sum by the projected area of the spacecraft alone to attain the total volume swept prior to \\
& deployment. \\
& Calculate the sum of the distances calculated in step 3 after deployment of drag augmentation device and \\
& multiply that sum by the projected area of the spacecraft with the device deployed to attain the volume swept \\
& after deployment. \\
& Calculate the total volume swept over the orbit lifetime by summing the volumes swept prior-to and after
\end{tabular}


as beginning when the spacecraft is fully decommissioned and passivated. End-of-life is defined as the point at which the spacecraft no longer exists, due to re-entry in the Earth's atmosphere, taken within this article as $65 \mathrm{~km}$ altitude.

Principal results are presented for a hypothetical scenario involving UKube-1 (COSPAR identification 2014037F), a three-unit, or 3U CubeSat with three deployable solar panels that has a mass of $3.98 \mathrm{~kg}$. As of September 9, 2016, UKube-1 has been inactive and believed to be tumbling randomly [27], hence at end-of-mission as defined herein. On this date, the spacecraft had a semi-major axis of $7006.23 \mathrm{~km}$, eccentricity of 0.0003369 , and inclination of $98.4032^{\circ}$ [28]. Note that the spacecraft mass is a measured quantity, and the semi-major axis, eccentricity, and inclination are taken from orbit tracking data, and as such each are specified to the level of detail available. If UKube-1 is considered randomly tumbling its projected area is calculated using the method of area-averaging outlined in the ISO standard for orbit lifetime estimation [25], found to be $0.0628 \mathrm{~m}^{2}$. The drag coefficient of spacecraft is also based on the ISO standard for orbit lifetime estimation, giving an assumed drag coefficient of 2.2 [25].

Secondary results are presented in the discussion section for CanX-7 (COSPAR identification 2016-059F), a $3 \mathrm{U}$ CubeSat $(34 \mathrm{~cm} \times 10 \mathrm{~cm} \times 10 \mathrm{~cm})$ of mass $3.5 \mathrm{~kg}$, with a drag sail of effective area of $2 \mathrm{~m}^{2}$ deployed on May 3, 2017 [29]. The initial epoch of the CanX-7 case study is May 4, 2017, when the spacecraft had a semimajor axis of $7059.3238 \mathrm{~km}$, eccentricity of 0.0030913 , and inclination of $98.1796^{\circ}$ [28]. Once again, the mass is a referenced value, while the semi-major axis, eccentricity and inclination are taken from orbit tracking data, and as such are specified to the level of detail available. If CanX-7 is considered randomly tumbling, without the drag sail deployed, its projected area is calculated to be $0.039 \mathrm{~m}^{2}$. The drag sail is assumed to stabilize the attitude of spacecraft such that it projects the sail effective area to the atmosphere [29].

\section{$3 \quad$ Results}

To demonstrate the effect of a time varying atmosphere the UKube-1 spacecraft is used as a case study. Alongside an analysis of the likely behavior of UKube-1, two hypothetical cases are considered, both assuming that
UKube-1 has a drag augmentation device of projected area $10 \mathrm{~m}^{2}$ on-board. The first hypothetical case assumes this device is deployed immediately at end-of-mission, while the second considers what happens when the deployment is delayed in order to coincide with the subsequent period of high solar activity. Figure 2(a) shows the orbit lifetime for each case, and Fig. 2(b) shows volume swept during each de-orbit scenario. Note that the analysis in Fig. 2 is for start epochs spaced evenly through an average solar cycle. Hence, it is not linked to the above end-of-mission epoch for UKube-1, providing an indicative in-sight rather than a prediction. It is noted that the current solar activity cycle is nearly over. Predicting the behavior of the subsequent cycle is extremely difficult; a cycle behavior can, currently, only be accurately predicted once it has begun, and even then, estimates can vary vastly due to the chaotic nature of the solar cycle. Therefore, an average cycle is used when predicting orbit

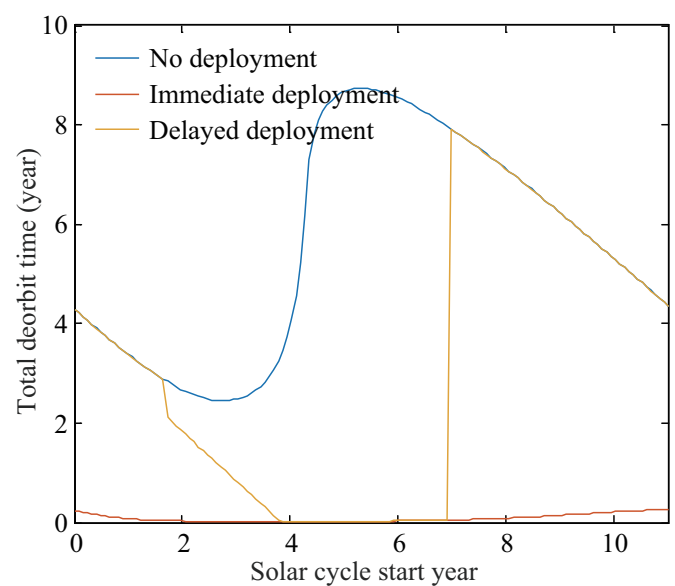

(a)

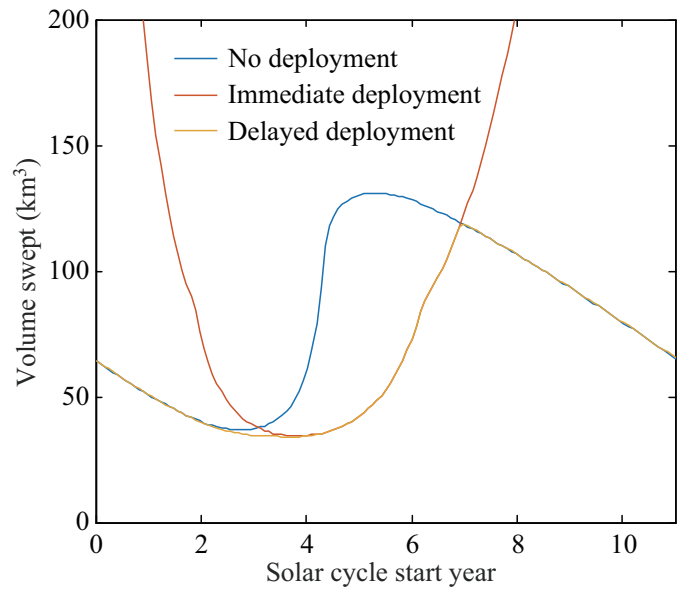

(b)

Fig. 2 UKube-1 de-orbit characteristics: (a) time and (b) volume swept, as affected by de-orbit start epoch through solar cycle. 
lifetime beyond the current cycle. This does introduce uncertainties on the order of magnitudes, providing an indicative insight rather than a prediction. Until solar cycle modelling becomes more accurate using an averaged cycle is the only prudent approach. The average cycle used herein was statistically derived from previous cycles and has a mean of 140 sfu. Further, the atmospheric density model used can have a significant impact on the orbit lifetime and, hence, volume swept. Herein, the analytical model developed by Kerr and Macdonald based on JB2008 is used [24]. JB2008 is the model recommended in the 2012 Committee on Space Research International Reference Atmosphere for total atmospheric mass density calculation [30]. Kerr and Macdonald developed an interpolated form of JB2008 and a density index allowing direct incorporation of solar activity [24].

In Fig. 2, "no deployment" denotes the case of decaying naturally due to atmospheric friction assuming random tumbling with no de-orbit device. "Immediate deployment" and "delayed deployment" denote the hypothetical cases of a $10 \mathrm{~m}^{2}$ drag augmentation device. The solar activity cycle start years of zero and five correspond to the average solar activity cycle minimum and maximum respectively.

Figure 2 shows that the date during the solar activity cycle, at which de-orbit begins, has a significant effect on both the de-orbit lifetime and the volume swept during de-orbit. A minimum in both can be observed approximately 3-5 years into the solar activity cycle, just prior to the maximum of the solar activity cycle. Figure 2(b) has been truncated to highlight the detail. However, in the full figure it could be seen that if deployment occurs at approximately year 4 , the volume swept is an order of magnitude lower than if deployment occurred at the solar activity cycle minimum (year 0/year 11). Thus, while orbit lifetime is always reduced, often drastically, by introducing a drag augmentation device, the volume swept is often increased. Drag augmentation should therefore only be used during, or just prior to, the maximum of a solar activity cycle. However, end-of-mission cannot always be predicted or guaranteed to coincide with this period. Therefore, when using a drag augmentation device, it should be capable of delaying deployment from the end-of-mission and spacecraft decommissioning to ensure that deployment coincides with the maximum of the upcoming solar activity cycle. Hence, the third case considered, explicitly detailed in Table 2, where the spacecraft decays naturally due to atmospheric friction on the tumbling spacecraft's projected area alone until the appropriate deployment date, at which time the drag augmentation device is deployed. The appropriate date is mission specific. Thus, the likely volume swept can be calculated using a Monte Carlo analysis with the deployment date during the solar cycle as the variable. In comparison to the "immediate deployment" case, except for the period around solar maximum, the de-orbit time increases, but the volume swept is considerably reduced. As shown in Fig. 2(a), both the "immediate deployment" and "delayed deployment" cases decrease or maintain the de-orbit lifetime, compared to the "no deployment" case. It is also noted in this figure that the time for delayed deployment significantly increases at approximately year seven of the solar cycle as this is the latest point that the deployment can be made within the cycle, beyond this point deployment must be delayed until after the next solar minimum. Resulting in a step-change in deorbit time. It is concluded that delayed deployment is a satisfactory compromise.

As the method is semi-analytical, see Table 2, the identified trends will be mathematically consistent with reduced duration and/or altitude variation case studies. For example, considering only the period between two altitude bands rather than until de-orbit is complete, this means that the identified trends will be consistent through regions of dense debris, as well as regions of sparse debris. However, considering the current space object population can provide further insight. Figure 3 shows a histogram of the percentage of regularly tracked objects from the current catalogue in $100 \mathrm{~km}$ altitude bins ${ }^{\S}$.

It can be seen in Fig. 3 that around $60 \%$ of the catalogued objects are at altitudes in the range $700-1000 \mathrm{~km}$. Ideally, an object would spend as little time, and sweep as little volume as possible in these highly populated regions during the de-orbit phase. By calculating the volume swept by a spacecraft in each region, a weighted metric is proposed that considers the volume swept through more populated regions as a greater risk than the same volume swept in a region with a lower percentage population of objects. This allows operators to consider this increased risk during initial mission design. Figure 4 shows the volume swept by UKube-1 in each region, (a) without and (b) with a drag sail deployed.

$\S$ As given in the Space-Track TLE catalogue, obtained from www. space-track.org on July 06, 2020. 


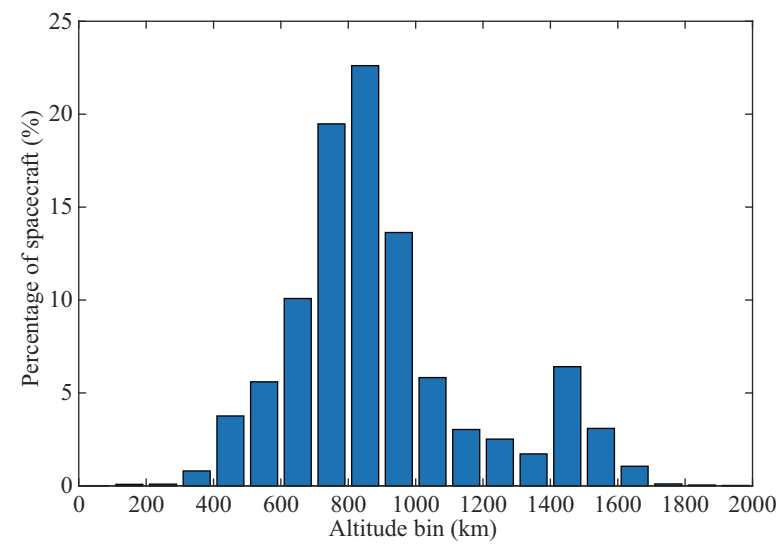

Fig. 3 Population of objects in LEO separated into $100 \mathrm{~km}$ altitude bins.
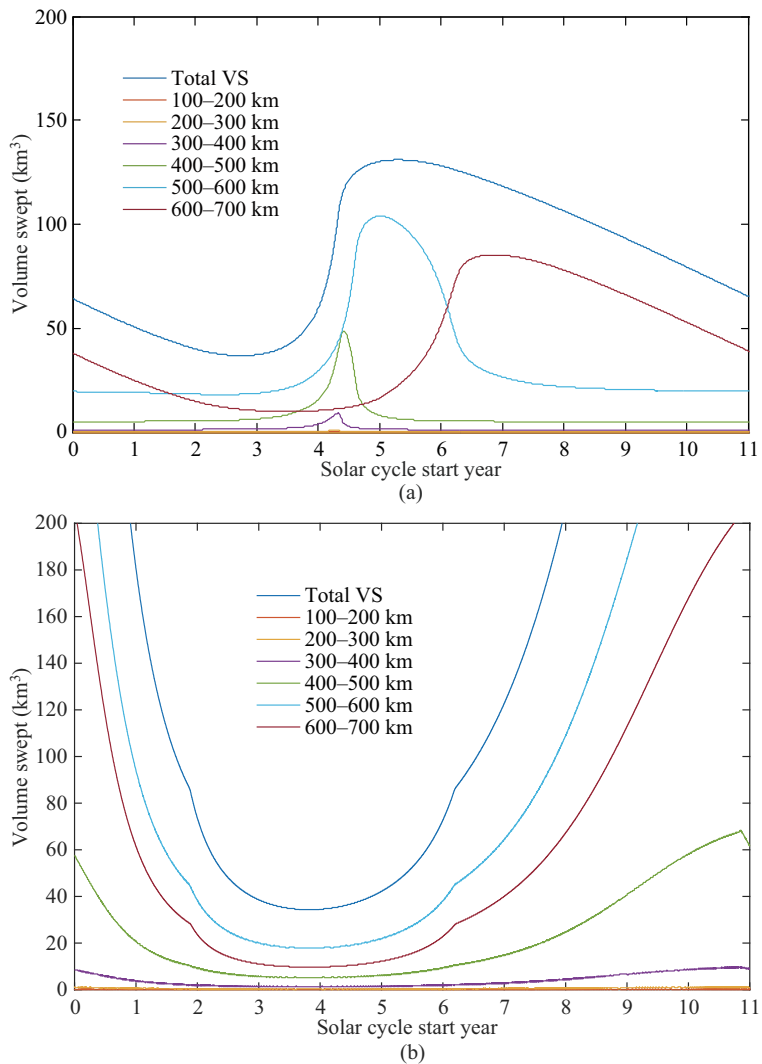

Fig. 4 Volume swept by UKube-1 during deorbit in different regions: (a) without and (b) with $10 \mathrm{~m}^{2}$ drag sail deployed, as affected by deorbit start date through solar cycle.

UKube-1 has a relatively low initial altitude so it spends no time in the highly populated regions, between 700 and $1000 \mathrm{~km}$. With the exception of the first altitude bin, UKube-1 will sweep less volume in each successive bin as altitude loss accelerates due to the increasing atmospheric drag force. The volume swept in the first altitude bin is dependent on the initial altitude within that bin. UKube1 began the de-orbit phase at approximately $628 \mathrm{~km}$ altitude, consequently less volume is swept in the 600 $700 \mathrm{~km}$ region than in the $500-600 \mathrm{~km}$ region. Note that a similar rational explains the different shape of the line in Fig. 4(a) for the 600-700 km region in comparison to other altitude bins. This is primarily driven by the orbit lifetime and initial altitude; with de-orbit beginning at or just after a solar maximum and failing to complete prior to solar minimum, resulting in an increased volume being swept until solar activity again increases. The peak of this initial altitude bin is displaced as the spacecraft only transits, approximately, the bottom quarter of it.

Using the population density in each region, a weighted volume swept is calculated as

$$
\mathrm{VS}_{\mathrm{PW}}=\mathrm{VS}\left(\frac{P+N}{N}\right)
$$

where $\mathrm{VS}_{\mathrm{PW}}$ is the population weighted volume swept, VS is the volume swept, $P$ is the number of objects in the region of interest, and $N$ is the total number of objects. This formulation provides a concise means to represent the local density of objects in a given region of space, providing a means of assessing collision risk. Figure 5 shows the volume swept by UKube- 1 with and without the population weighting, denoted by "integrated risk".

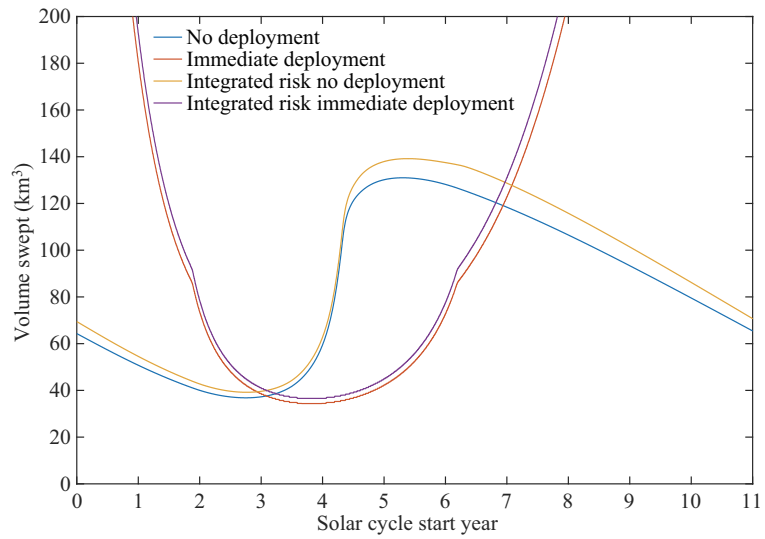

Fig. 5 Volume swept by UKube-1 (with and without integrated risk) during de-orbit as affected by de-orbit start date through solar cycle.

It can be seen in Fig. 5 that the volume swept in both cases with the integrated risk is higher, as expected. However, the volume swept is not significantly altered in either case. This is primarily due to the low initial altitude of UKube-1, affording it a safer de-orbit overall. Figures 4 and 5 have been reproduced in Figs. 6 and 7, using the hypothetical case of UKube-1 having an initial 

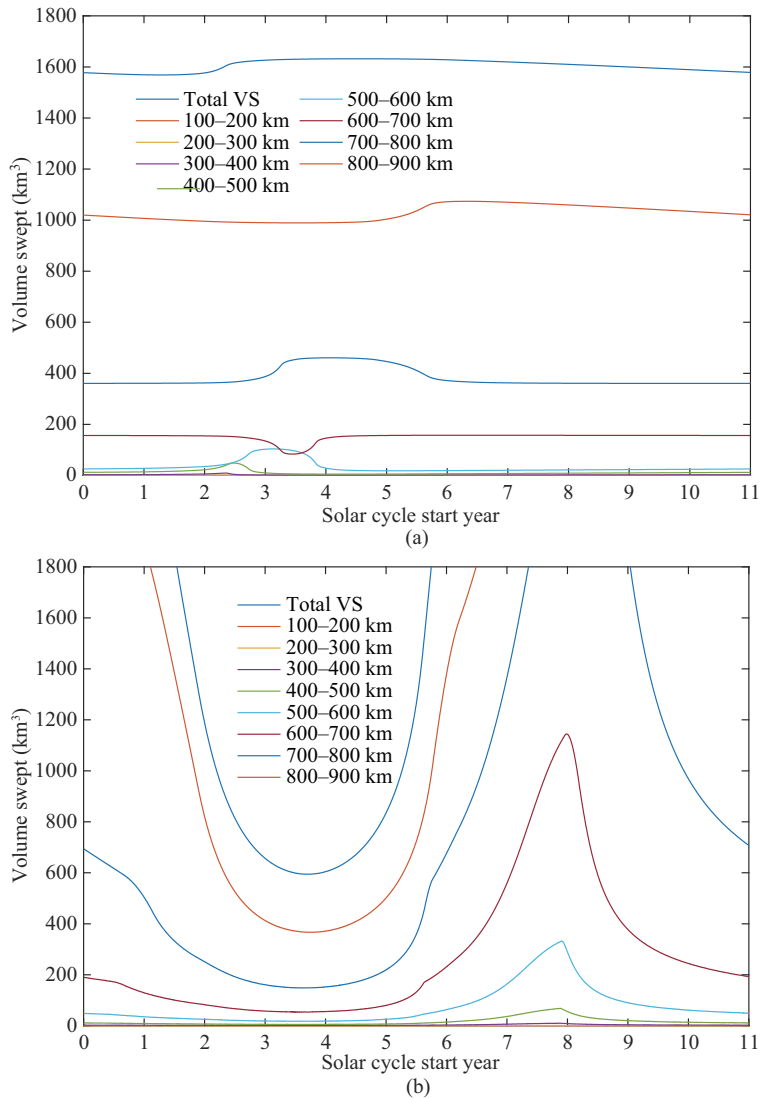

Fig. 6 Volume swept by UKube-1 (initial altitude $900 \mathrm{~km}$ ) during deorbit in different regions: (a) without and (b) with $10 \mathrm{~m}^{2}$ drag sail deployed, as affected by deorbit start date through solar cycle.

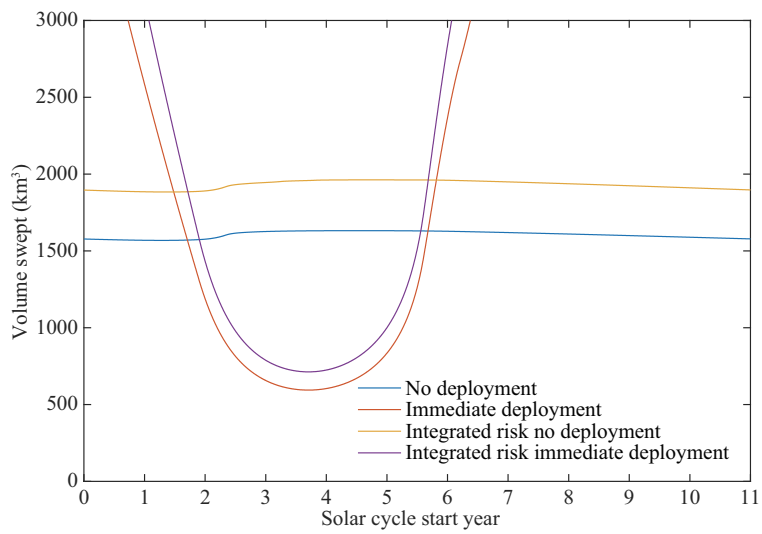

Fig. 7 UKube-1 (initial altitude $900 \mathrm{~km}$ ) volume swept, with and without integrated risk, during de-orbit as affected by de-orbit start date through solar cycle.

altitude of $900 \mathrm{~km}$ to show the result of passing through a more populated region.

It can be seen in Figs. 6 and 7 that the impact of the weighting is more prominent for a case with a higher initial altitude. In comparing the untruncated versions of Figs. 5 and 7 , it could be more clearly seen for the higher initial altitude, that if the spacecraft deploys the drag sail at the least opportune moment during the solar activity cycle (around 8-9 years through the solar activity cycle) that the effect of the weighting is greater than if the solar activity cycle is exploited (with sail deployment occurring at 3-5 years). This indicates that the worstcase scenario introduces more risk than can be determined by than volume swept alone, as the larger volume is being swept out of the most populated regions. Furthermore, the ideal deployment window (specifically, the window when the immediate deployment volume swept is less than the no deployment volume swept) is reduced, and thus the possibility of a random failure occurring at an inopportune moment is increased. Thus, reinforcing the argument for a delayed deployment capability.

\section{Discussion}

Several major consequences of delayed deployment should be considered. First, increasing the time to deorbit potentially increases the risk of an unplanned break-up. The challenge of designing a sub-system that must remain idle for up to eight years, after end-of-mission, then assuredly activate and deploy a drag augmentation device from a tumbling spacecraft will also potentially increase the failure risk rate and/or system complexity, and hence likely cost. The lack of complexity and low sub-system cost are the current attractors of the drag augmentation concept. The outcome of any collision should also be considered. Although the volume swept is increased by the introduction of a drag augmentation device, a collision with the thin film of the sail area is less likely to cause catastrophic damage. Therefore, although the collision risk increases, a directly proportional increase in liability risk cannot be assumed as this would require a statistical analysis of the space object population coupled with the probability of the object being de-orbited, with and without a drag augmentation device, having a conjunction with another space object from that population. However, in not implementing the delayed deployment method operators tacitly accept the potential for an increase in volume swept and hence the increased risk of a collision occurrence, catastrophic or not.

Noting that on-orbit liability is fault-based, a terrestrial analogy can be made. In 1947, the United States of America Court of Appeals for the Second Circuit proposed a test to determine the standard of care for the tort 
of negligence, this was the first-time calculus was used to determine liability. The judgment was written by Judge Learned Hand wherein he described what is now termed the calculus of negligence, or the Learned Hand formula, an algebraic formula, $B=P \cdot L$. From this, liability is based on the relation between investment in precaution $B$ and the product of the probability $P$ and magnitude $L$ of resulting harm. Where $P \cdot L$ exceeds $B$, the defendant should be liable, where $B$ is equal to or greater than $P \cdot L$, the defendant should not be held liable [31]. The application of the Learned Hand formula in outer space has not been much discussed; however by increasing the probability of a collision the Learned Hand formula implies a reckless act, in which case spacecraft operators who inappropriately deploy a drag augmentation device, and hence the State Party that licensed the operation, could be argued liable for any subsequent on-orbit collision. Depending on the outcome of a collision an argument could be made to limit liability, as the introduction of an inappropriately deployed drag augmentation device would have increased the probability, $P$, but may also have decreased the magnitude of resultant harm, $L$. This assumes that collision occurred with the sail area rather than the main spacecraft body, resulting in, likely, less than catastrophic damage. However, as the collision is an unpredictable event, the only responsible path for space actors, and those who license them, is to reduce the probability of any type of collision occurring in the first place.

Of note, the technology demonstration mission CanX7 is currently on-orbit and deployed a drag sail in May 2017 [29], just ahead of a solar minimum, after the end of its operational life. In doing so the operators of CanX7 have, perhaps unwittingly, increased the on-orbit collision risk of the spacecraft. Figure 8 recreates the analysis completed in the prior section for UKube-1, for CanX-7. It shows the effect of varying the epoch through the solar activity cycle at which de-orbit begins on the de-orbit lifetime and the volume swept during de-orbit. Note that once again the analysis in Fig. 8 is for start epochs spaced evenly through an average solar cycle, and is hence not linked to the actual deployment date. However, note the addition of the vertical line showing the actual deployment epoch of the CanX-7 drag sail. As Fig. 8 assumes an average solar cycle it gives only an indicative in-sight rather than a prediction.
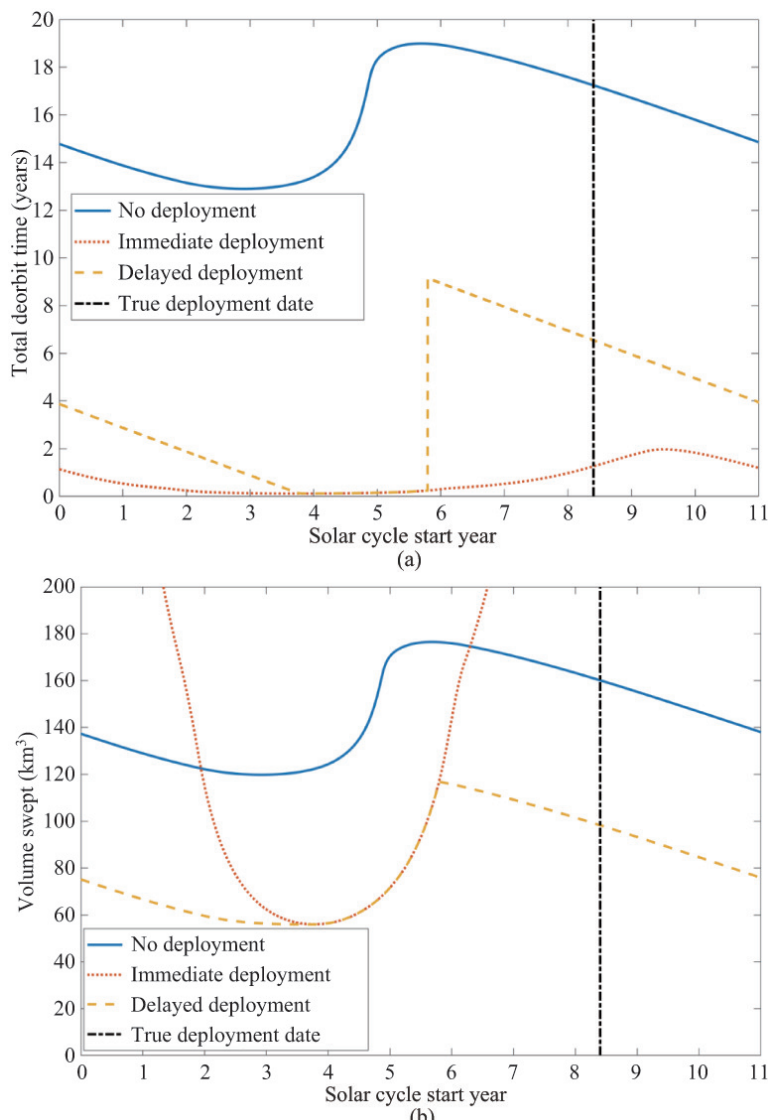

Fig. 8 CanX-7 deorbit characteristics, (a) time and (b) volume swept, as affected by de-orbit start epoch through solar cycle.

In comparison to the UKube-1 case study, delayed deployment now shows a marked improvement over the no deployment cases. This is due to the duration of the no deployment case exceeding a single solar cycle, as such the delayed deployment provides a much shorter lifetime. A resultant smaller volume swept is also realised. CanX-7 deployed its drag sail at an inopportune moment, in a non-truncated version of Fig. 8(b) it could be seen that during the theoretical average solar cycle used herein the deployment on May 3, 2017 produces an estimated volume swept of $\sim 605 \mathrm{~km}^{3}$ in comparison to the $\sim 160$ or $\sim 98 \mathrm{~km}^{3}$ produced in the no deployment and delayed deployment cases respectively for that date. Ideally, given that CanX-7 was a technology demonstrator mission primarily designed to demonstrate a drag sail, the operators of CanX-7 should have delayed its launch for around 6.5 years, and then launched and performed immediate deployment during November 2023. This scenario would have produced a total volume swept of $\sim 56 \mathrm{~km}^{3}\left(\sim 55 \mathrm{~km}^{3}\right.$ by the sail and $\sim 1 \mathrm{~km}^{3}$ by the 
spacecraft body) based on the average solar cycle data used herein. If they had done so CanX-7 would have swept the least total volume possible, minimizing both the risk of a collision occurrence and the risk posed by that collision.

It is important to note that the mitigation strategy of delayed deployment is most effective if the drag augmentation device is of sufficient size to cause the spacecraft to de-orbit over one solar activity maximum period. The device may therefore have to be larger than would be required to de-orbit over the minimum required period of 25 years given by the debris mitigation guidelines.

Finally, one unquantifiable risk, not considered herein, is that by delaying deployment, and therefore increasing time on-orbit, an increased on-orbit population could be encountered at the time of deployment. Thus, the collision, or integrated risk would also be increased. However, there is no way to accurately predict future populations and regulatory practice focuses on the current situation, and the effect of any action on existing space actors and objects.

\section{Conclusions}

Rather than reduce the risk of an on-orbit collision occurring, as is widely held, the orbit removal concept known as drag augmentation often increases the risk. This increase is induced by the solar activity cycle, which causes Earth's atmosphere to be time-variant and dynamic. Drag augmentation should therefore ideally only be used during, or just prior to, the maximum of a solar activity cycle. However, end-of-mission cannot be guaranteed to coincide with this period. Therefore, when using a drag augmentation device, it should be capable of delaying deployment from the end-of-mission and spacecraft decommissioning for up to eight years and should be able to deploy from a tumbling, otherwise passivated spacecraft. This new requirement likely increases system complexity, and hence cost; the lack of complexity and low-cost being the current attractors of the concept. Additionally, the drag surface should be sized to ensure de-orbit is completed in one solar cycle rather than to comply with the 25 -year best-practice guidelines. Noting that such devices have already been deployed into low-Earth orbit, and that the Outer Space Treaty requires "continuing supervision by the appropriate State Party", application of the Learned Hand formula implies spacecraft operators who plan to inappropriately deploy a drag augmentation device, and hence the State Party that licensed them, could be argued liable for any subsequent on-orbit collision. Therefore, it is recommended that these licensing authorities consider prior, and any future approval to deploy such devices as that State Party could bear international responsibility for any subsequent collision.

\section{Acknowledgements}

The authors thank Dr. Lesley Jane Smith of Leuphana University of Lüneburg for her discussions on the use of the Learned Hand formula with regard to liability in space. This work received funding from the European Union's Horizon 2020 research and innovation program under grant agreement No. 687295.

\section{References}

[1] Kelso, T. S. Analysis of the 2007 Chinese ASAT test and the impact of its debris on the space environment. In: Proceedings of the 8th Advanced Maui Optical and Space Surveillance Technologies Conference, 2007: 321-330.

[2] Kelso, T. S. Analysis of the Iridium 33-Cosmos 2251 collision. In: Proceedings of the 19th AIAA/AAS Astrodynamics Specialist Conference, 2009: AAS 09-368.

[3] Heidt, H., Puig-Suari, J., Moore, A., Nakasuka, S., Twiggs, R. CubeSat: A new generation of picosatellite for education and industry low-cost space experimentation. In: Proceedings of the 14th AIAA/USU Conference on Small Satellites, 2000: SSC01-VIIIb-5.

[4] National Academies of Sciences, Engineering, and Medicine. Achieving Science with CubeSats: Thinking inside the Box. Washington, D.C.: National Academies Press, 2016.

[5] United Nations Committee on the Peaceful Uses of Outer Space Legal Subcommittee. Treaty on principles governing the activities of states in the exploration and use of outer space, including the Moon and other celestial bodies, 1966.

[6] United Nations Committee on the Peaceful Uses of Outer Space Legal Subcommittee. Convention on international liability for damage caused by space objects, 1971 .

[7] MacDonald, M., Badescu, V. The International Handbook of Space Technology. Berlin, Heidelberg: Springer Berlin Heidelberg, 2014.

[8] International Organization for Standardization. ISO 24113: 2011 space systems - space debris mitigation requirements, 2011.

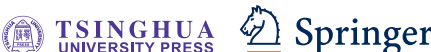


[9] Inter-Agency Space Debris Coordination Committee. IADC-02-01 Space Debris Mitigation Guidelines, 2007: 1-10.

[10] Kerr, E., Macdonald, M., Voigt, P. Taxonomy and analysis of issues facing post-mission disposal concepts. In: Proceedings of the 68th International Astronautical Congress, 2017: 3735-3744.

[11] Information on https://www.gov.uk/government/news/ threetwo-one-blast-off-dstl-launches-50-million-spaceprogramme (cited 11 July 2017).

[12] Nock, K. T., Aaron, K. M., McKnight, D. Removing orbital debris with less risk. Journal of Spacecraft and Rockets, 2013, 50(2): 365-379.

[13] Stohlman, O. R., Lappas, V. Deorbitsail: a deployable sail for de-orbiting. In: Proceedings of the 54th AIAA/ASME/ASCE/AHS/ASC Structures, Structural Dynamics, and Materials Conference, 2013: AIAA 2013-1806.

[14] Fernandez, J. M., Rose, G. K., Younger, C. J., Dean, G. D., Warren, J. E., Stohlman, O. R., Wilkie, W. K. NASA's advanced solar sail propulsion system for low-cost deep space exploration and science missions that uses high performance rollable composite booms. In: Proceedings of the 4th International Symposium on Solar Sailing, 2017.

[15] Visagie, L., Lappas, V., Erb, S. Drag sails for space debris mitigation. Acta Astronautica, 2015, 109: 65-75.

[16] Forshaw, J. L., Aglietti, G. S., Navarathinam, N., Kadhem, H., Salmon, T., Pisseloup, A., Joffre, E., Chabot, T., Retat, I., Axthelm, R. et al. RemoveDEBRIS: An in-orbit active debris removal demonstration mission. Acta Astronautica, 2016, 127: 448-463.

[17] Forshaw, J. L., Aglietti, G. S., Salmon, T., Retat, I., Roe, M., Burgess, C., Chabot, T., Pisseloup, A., Phipps, A., Bernal, C., et al. Final payload test results for the RemoveDebris active debris removal mission. Acta Astronautica 2017, 138: 326-342.

[18] Guglielmo, D., Omar, S., Bevilacqua, R. Drag de-orbit device: A new standard reentry actuator for CubeSats. Journal of Spacecraft and Rockets, 2018, https://doi. org/10.2514/1.A34218.

[19] Werner, D. Drag sails could counter debris. Aerospace America, 2017.

[20] Information on http://news.bbc.co.uk/1/hi/8590103.stm (cited 11 July 2018).

[21] Information on https://www.bbc.co.uk/news/scienceenvironment-43584070 (cited 11 July 2018).

[22] Ahmadloo, H., Zhang, J. De-orbiting collision risk assessment and detailed orbital simulation of LEO space debris removal drag sail. In: Proceedings of the 9th Asian-Pacific Conference on Aerospace and Science/the 2nd Asian Joint Symposium on Aerospace Engineering, 2017: 201-215.

[23] Colombo, C., Rossi, A., Dalla Vedova, F., Francesconi, A., Bombardelli, C., Trisolini, M., Gonzalo, J. L., Di Lizia, P., Giacomuzzo, C., Khan, S. B., et al. Effects of passive de-orbiting through drag and solar sails and electrodynamic tethers on the space debris environment. In: Proceedings of the 6th International Astronautical Congress, 2018: IAC-18-A6.2.8.

[24] Kerr, E., MacDonald, M. Incorporating solar activity into general perturbation analysis of atmospheric friction. Journal of Guidance, Control, and Dynamics, 2018, 41(6): 1320-1336.

[25] International Organization for Standardization. ISO 27852:2016: Space systems - Estimation of orbit lifetime, 2016.

[26] Vasile, M. L., Minisci, E., Serra, R., Beck, J., Holbrough, I. Analysis of the de-orbiting and re-entry of space objects with high area to mass ratio. In: Proceedings of the AIAA/AAS Astrodynamics Specialist Conference, AIAA, 2016: AIAA 2016-5678.

[27] Walker, H. UKube-1: operations and lessons learned. In: Proceedings of the 8th European CubeSat Symposium, 2016.

[28] Information on http://celestrak.com/ (cited 11 July 2018).

[29] Cotton, B., Bennett, I., Zee, R. E. On-orbit results from the CanX-7 drag sail deorbit mission. In: Proceedings of the 31st Annual AIAA/USU Small Satellite Conference, 2017.

[30] Commitee on Space Research. COSPAR International Reference Atmosphere, 2012.

[31] Grossman, P. Z., Cearley, R. W., Cole, D. H. Uncertainty, insurance and the Learned Hand formula. Law, Probability and Risk, 2006, 5(1): 1-18.

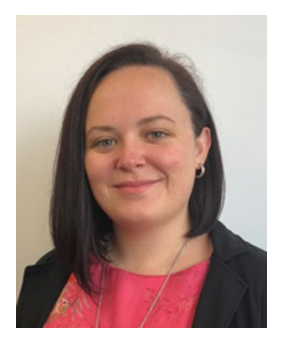

Emma Kerr is currently working as a space safety engineer and project manager for Deimos Space UK Ltd. She received her Ph.D. degree in aerospace engineering from the University of Strathclyde, UK, where she also worked as a research assistant. Following her Ph.D. degree, Emma worked as a post-doctoral fellow for the Space Environment Research Centre based at RMIT University, Australia, specialising in space weather and atmospheric density effects on orbit propagation.

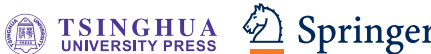




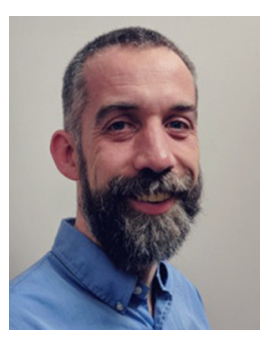

Malcolm Macdonald is professor and chair of applied space technology at University of Strathclyde, working at the interface between academia, industry, and government. His work aims to enable and develop new spacederived services through advancing a range of new technologies, challenging conventional ideas, and working at the interface between disciplines to advance new concepts in the exploration and exploitation of space. He is a fellow of the Royal Aeronautical Society, and an associate fellow of the AIAA. E-mail: Malcolm.macdonald.102@strath.ac.uk.

Open Access This article is licensed under a Creative Com- mons Attribution 4.0 International License, which permits use, sharing, adaptation, distribution and reproduction in any medium or format, as long as you give appropriate credit to the original author(s) and the source, provide a link to the Creative Commons licence, and indicate if changes were made.

The images or other third party material in this article are included in the article's Creative Commons licence, unless indicated otherwise in a credit line to the material. If material is not included in the article's Creative Commons licence and your intended use is not permitted by statutory regulation or exceeds the permitted use, you will need to obtain permission directly from the copyright holder.

To view a copy of this licence, visit http:// creativecommons.org/licenses/by/4.0/. 